\title{
Learning Semantic Links from a Corpus of Parallel Temporal and Causal Relations
}

\author{
Steven Bethard \\ Institute for Cognitive Science \\ Department of Computer Science \\ University of Colorado \\ Boulder, CO 80309, USA \\ steven. bethardecolorado. edu
}

\author{
James H. Martin \\ Institute for Cognitive Science \\ Department of Computer Science \\ University of Colorado \\ Boulder, CO 80309, USA \\ james.martin@colorado.edu
}

\begin{abstract}
Finding temporal and causal relations is crucial to understanding the semantic structure of a text. Since existing corpora provide no parallel temporal and causal annotations, we annotated 1000 conjoined event pairs, achieving inter-annotator agreement of $81.2 \%$ on temporal relations and $77.8 \%$ on causal relations. We trained machine learning models using features derived from WordNet and the Google N-gram corpus, and they outperformed a variety of baselines, achieving an F-measure of 49.0 for temporals and 52.4 for causals. Analysis of these models suggests that additional data will improve performance, and that temporal information is crucial to causal relation identification.
\end{abstract}

\section{Introduction}

Working out how events are tied together temporally and causally is a crucial component for successful natural language understanding. Consider the text:

(1) I ate a bad tuna sandwich, got food poisoning and had to have a shot in my shoulder. wsj_0409

To understand the semantic structure here, a system must order events along a timeline, recognizing that getting food poisoning occurred BEFORE having a shot. The system must also identify when an event is not independent of the surrounding events, e.g. got food poisoning was CAUSED by eating a bad sandwich. Recognizing these temporal and causal relations is crucial for applications like question answering which must face queries like How did he get food poisoning? or What was the treatment?
Currently, no existing resource has all the necessary pieces for investigating parallel temporal and causal phenomena. The TimeBank (Pustejovsky et al., 2003) links events with BEFORE and AFTER relations, but includes no causal links. PropBank (Kingsbury and Palmer, 2002) identifies ARGM-TMP and ARGM-CAU relations, but arguments may only be temporal or causal, never both. Thus existing corpora are missing some crucial pieces for studying temporal-causal interactions. Our research aims to fill these gaps by building a corpus of parallel temporal and causal relations and exploring machine learning approaches to extracting these relations.

\section{Related Work}

Much recent work on temporal relations revolved around the TimeBank and TempEval (Verhagen et al., 2007). These works annotated temporal relations between events and times, but low inter-annotator agreement made many TimeBank and TempEval tasks difficult (Boguraev and Ando, 2005; Verhagen et al., 2007). Still, TempEval showed that on a constrained tense identification task, systems could achieve accuracies in the 80s, and Bethard and colleagues (Bethard et al., 2007) showed that temporal relations between a verb and a complement clause could be identified with accuracies of nearly $90 \%$.

Recent work on causal relations has also found that arbitrary relations in text are difficult to annotate and give poor system performance (Reitter, 2003). Girju and colleagues have made progress by selecting constrained pairs of events using web search patterns. Both manually generated Cause-Effect patterns (Girju et al., 2007) and patterns based on nouns 


\begin{tabular}{l|rrr} 
& Full & Train & Test \\
\hline \hline Documents & 556 & 344 & 212 \\
Event pairs & 1000 & 697 & 303 \\
\hline BEFORE relations & 313 & 232 & 81 \\
AFTER relations & 16 & 11 & 5 \\
CAUSAL relations & 271 & 207 & 64
\end{tabular}

Table 1: Contents of the corpus and its train/test sections

\begin{tabular}{l|ccc} 
Task & Agreement & Kappa & $\mathrm{F}$ \\
\hline \hline Temporals & 81.2 & 0.715 & 71.9 \\
Causals & 77.8 & 0.556 & 66.5
\end{tabular}

Table 2: Inter-annotator agreement by task.

linked causally in WordNet (Girju, 2003) were used to collect examples for annotation, with the resulting corpora allowing machine learning models to achieve performance in the 70s and 80s.

\section{Conjoined Events Corpus}

Prior work showed that finding temporal and causal relations is more tractable in carefully selected corpora. Thus we chose a simple construction that frequently expressed both temporal and causal relations, and accounted for $10 \%$ of all adjacent verbal events: events conjoined by the word and.

Our temporal annotation guidelines were based on the guidelines for TimeBank and TempEval, augmented with the guidelines of (Bethard et al., 2008). Annotators used the labels:

BEFORE The first event fully precedes the second AFTER The second event fully precedes the first NO-REL Neither event clearly precedes the other

Our causal annotation guidelines were based on paraphrasing rather than the intuitive notions of cause used in prior work (Girju, 2003; Girju et al., 2007). Annotators selected the best paraphrase of "and" from the following options:

CAUSAL and as a result, and as a consequence, and enabled by that

NO-REL and independently, and for similar reasons

To build the corpus, we first identified verbs that represented events by running the system of (Bethard and Martin, 2006) on the TreeBank. We then used a set of tree-walking rules to identify conjoined event pairs. 1000 pairs were annotated by two annotators and adjudicated by a third. Table 1

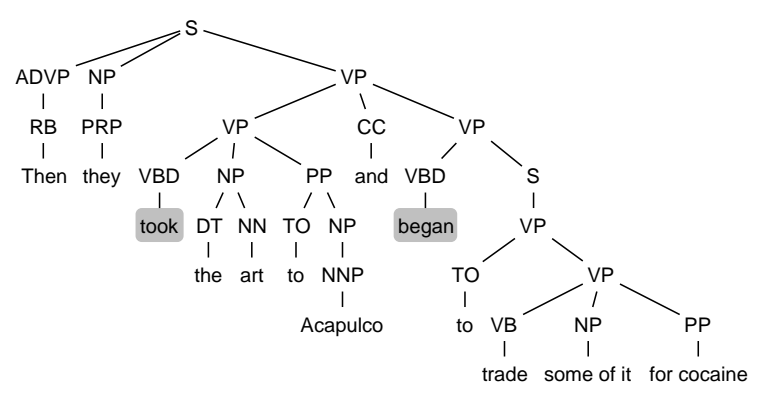

Figure 1: Syntactic tree from wsj_0450 with events took and began highlighted.

and Table 2 give statistics for the resulting corpus ${ }^{1}$. The annotators had substantial agreement on temporals $(81.2 \%)$ and moderate agreement on causals (77.8\%). We also report F-measure agreement, since BEFORE, AFTER and CAUSAL relations are more interesting than NO-REL. Annotators had F-measure agreement of 71.9 on temporals and 66.5 causals.

\section{Machine Learning Methods}

We used our corpus for machine learning experiments where relation identification was viewed as pair-wise classification. Consider the sentence:

(2) The man who had brought it in for an estimate had [EVENT returned] to collect it and was [EVENT waiting] in the hall. wsj_0450

A temporal classifier should label returned-waiting with BEFORE since returned occurred first, and a causal classifier should label it CAUSAL since this and can be paraphrased as and as a result.

We identified both syntactic and semantic features for our task. These will be described using the example event pair in Figure 1. Our syntactic features characterized surrounding surface structures:

- The event words, lemmas and part-of-speech tags, e.g. took, take, VBD and began, begin, VBD.

- All words, lemmas and part-of-speech tags in the verb phrases of each event, e.g. took, take, VBD and began, to, trade, begin, trade, $\mathrm{VBD}, \mathrm{TO}, \mathrm{VB}$.

- The syntactic paths from the first event to the common ancestor to the second event, e.g. $\mathrm{VBD}>\mathrm{VP}, \mathrm{VP}$ and $\mathrm{VP}<\mathrm{VBD}$.

\footnotetext{
${ }^{1}$ Train: wsj_0416-wsj_0759. Test: wsj_0760-wsj_0971. verbs.colorado.edu/ bethard/treebank-verb-conj-anns.xml
} 
- All words before, between and after the event pair, e.g. Then, they plus the, art, to, Acapulco, and plus to, trade, some, of, it, for, cocaine.

Our semantic features encoded surrounding word meanings. We used WordNet (Fellbaum, 1998) root synsets (roots) and lexicographer file names (lexnames) to derive the following features:

- All event roots and lexnames, e.g. take\#33, move\#1 ... body, change ... for took and be\#0, begin\#1 ... change, communication ... for began.

- All lexnames before, between and after the event pair, e.g. all plus artifact, location, etc. plus possession, artifact, etc.

- All roots and lexnames shared by both events, e.g. took and began were both act\#0, be\#0 and change, communication, etc.

- The least common ancestor (LCA) senses shared by both events, e.g. took and began meet only at their roots, so the LCA senses are $a c t \# 0$ and be\#0.

We also extracted temporal and causal word associations from the Google N-gram corpus (Brants and Franz, 2006), using <keyword> <pronoun> $<$ word > patterns, where before and after were the keywords for temporals, and because was the keyword for causals. Word scores were assigned as:

$$
\operatorname{score}(w)=\log \left(\frac{N_{\text {keyword }}(w)}{N(w)}\right)
$$

where $N_{\text {keyword }}(w)$ is the number of times the word appeared in the keyword's pattern, and $N(w)$ is the number of times the word was in the corpus. The following features were derived from these scores:

- Whether the event score was in at least the $N$ th percentile, e.g. took's -6.1 because score placed it above $84 \%$ of the scores, so the feature was true for $N=70$ and $N=80$, but false for $N=90$.

- Whether the first event score was greater than the second by at least $N$, e.g. took and began have after scores of -6.3 and -6.2 so the feature was true for $N=-1$, but false for $N=0$ and $N=1$.

\section{Results}

We trained SVM ${ }^{\text {perf }}$ classifiers (Joachims, 2005) for the temporal and causal relation tasks ${ }^{2}$ using the

\footnotetext{
${ }^{2}$ We built multi-class SVMs using the one-vs-rest approach and used 5-fold cross-validation on the training data to set parameters. For temporals, $\mathrm{C}=0.1$ (for syntactic-only models),
}

\begin{tabular}{l|rrr|rrr} 
& \multicolumn{3}{|c|}{ Temporals } & \multicolumn{3}{c}{ Causals } \\
Model & \multicolumn{1}{|c}{$\mathrm{P}$} & \multicolumn{1}{c}{$\mathrm{R} 1$} & $\mathrm{P}$ & $\mathrm{R}$ & $\mathrm{F} 1$ \\
\hline \hline BEFORE & 26.7 & 94.2 & 41.6 & - & - & - \\
CAUSAL & - & - & - & 21.1 & 100.0 & 34.8 \\
$1^{\text {st }}$ Event & 35.0 & 24.4 & 28.8 & 31.0 & 20.3 & 24.5 \\
$2^{\text {nd }}$ Event & 36.1 & 30.2 & 32.9 & 22.4 & 17.2 & 19.5 \\
POS Pair & 46.7 & 8.1 & 13.9 & 30.0 & 4.7 & 8.1 \\
\hline Syntactic & 36.5 & 53.5 & 43.4 & 24.4 & 79.7 & 37.4 \\
Semantic & 35.8 & 55.8 & 43.6 & 27.2 & 64.1 & 38.1 \\
All & 43.6 & 55.8 & $\mathbf{4 9 . 0}$ & 27.0 & 59.4 & 37.1 \\
All+Tmp & - & - & - & 46.9 & 59.4 & $\mathbf{5 2 . 4}$
\end{tabular}

Table 3: Performance of the temporal relation identification models: (A)ccuracy, (P)recision, (R)ecall and (F1)measure. The null label is NO-REL.

train/test split from Table 1 and the feature sets:

Syntactic The syntactic features from Section 4.

Semantic The semantic features from Section 4.

All Both syntactic and semantic features.

All+Tmp (Causals Only) Syntactic and semantic features, plus the gold-standard temporal label.

We compared our models against several baselines, using precision, recall and F-measure since the NOREL labels were uninteresting. Two simple baselines had 0\% recall: a lookup table of event word pairs ${ }^{3}$, and the majority class (NO-REL) label for causals. We therefore considered the following baselines:

BEFORE Classify all instances as BEFORE, the majority class label for temporals.

CAUSAL Classify all instances as CAUSAL.

$1^{\text {st }}$ Event Use a lookup table of $1^{\text {st }}$ words and the labels they were assigned in the training data.

$2^{\text {nd }}$ Event As $1^{\text {st }}$ Event, but using $2^{\text {nd }}$ words.

POS Pair As $1^{\text {st }}$ Event, but using part of speech tag pairs. POS tags encode tense, so this suggests the performance of a tense-based classifier.

The results on our test data are shown in Table 3. For temporal relations, the F-measures of all SVM models exceeded all baselines, with the combination of syntactic and semantic features performing 5 points better (43.6\% precision and 55.8\% recall) than either feature set individually. This suggests that our syntactic and semantic features encoded complementary information for the temporal relation task. For

$\mathrm{C}=1.0$ (for all other models), and loss-function=F1 (for all models). For causals, $\mathrm{C}=0.1$ and loss-function=precision/recall break even point (for all models).

${ }^{3}$ Only 3 word pairs from training were seen during testing. 


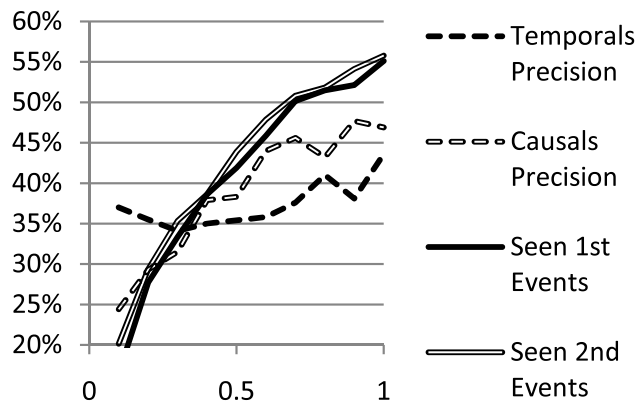

Figure 2: Model precisions (dotted lines) and percent of events in the test data seen during training (solid lines), given increasing fractions of the training data.

causal relations, all SVM models again exceeded all baselines, but combining syntactic features with semantic ones gained little. However, knowing about underlying temporal relations boosted performance to $46.9 \%$ precision and $59.4 \%$ recall. This shows that progress in causal relation identification will require knowledge of temporal relations.

We examined the effect of corpus size on our models by training them on increasing fractions of the training data and evaluating them on the test data. The precisions of the resulting models are shown as dotted lines in Figure 2. The models improve steadily, and the causals precision can be seen to follow the solid curves which show how event coverage increases with increased training data. A logarithmic trendline fit to these seen-event curves suggests that annotating all 5,013 event pairs in the Penn TreeBank could move event coverage up from the mid 50s to the mid 80s. Thus annotating additional data should provide a substantial benefit to our temporal and causal relation identification systems.

\section{Conclusions}

Our research fills a gap in existing corpora and NLP systems, examining parallel temporal and causal relations. We annotated 1000 event pairs conjoined by the word and, assigning each pair both a temporal and causal relation. Annotators achieved 81.2\% agreement on temporal relations and $77.8 \%$ agreement on causal relations. Using features based on WordNet and the Google N-gram corpus, we trained support vector machine models that achieved 49.0 $\mathrm{F}$ on temporal relations, and $37.1 \mathrm{~F}$ on causal relations. Providing temporal information to the causal relations classifier boosted its results to $52.4 \mathrm{~F}$. Fu- ture work will investigate increasing the size of the corpus and developing more statistical approaches like the Google N-gram scores to take advantage of large-scale resources to characterize word meaning.

\section{Acknowledgments}

This research was performed in part under an appointment to the U.S. Department of Homeland Security (DHS) Scholarship and Fellowship Program.

\section{References}

S. Bethard and J. H. Martin. 2006. Identification of event mentions and their semantic class. In EMNLP-2006.

S. Bethard, J. H. Martin, and S. Klingenstein. 2007. Timelines from text: Identification of syntactic temporal relations. In ICSC-2007.

S. Bethard, W. Corvey, S. Klingenstein, and J. H. Martin. 2008. Building a corpus of temporal-causal structure. In LREC-2008.

B. Boguraev and R. K. Ando. 2005. Timebankdriven timeml analysis. In Annotating, Extracting and Reasoning about Time and Events. IBFI, Schloss Dagstuhl, Germany.

T. Brants and A. Franz. 2006. Web 1t 5-gram version 1. Linguistic Data Consortium, Philadelphia.

C. Fellbaum, editor. 1998. WordNet: An Electronic Database. MIT Press.

R. Girju, P. Nakov, V. Nastase, S. Szpakowicz, P. Turney, and D. Yuret. 2007. Semeval-2007 task 04: Classification of semantic relations between nominals. In SemEval-2007.

R. Girju. 2003. Automatic detection of causal relations for question answering. In ACL Workshop on Multilingual Summarization and Question Answering.

T. Joachims. 2005. A support vector method for multivariate performance measures. In ICML-2005.

P. Kingsbury and M. Palmer. 2002. From Treebank to PropBank. In LREC-2002.

J. Pustejovsky, P. Hanks, R. Saurí, A. See, R. Gaizauskas, A. Setzer, D. Radev, B. Sundheim, D. Day, L. Ferro, and M. Lazo. 2003. The timebank corpus. In Corpus Linguistics, pages 647-656.

D. Reitter. 2003. Simple signals for complex rhetorics: On rhetorical analysis with rich-feature support vector models. LDV-Forum, GLDV-Journal for Computational Linguistics and Language Technology, 18(1/2):38-52.

M. Verhagen, R. Gaizauskas, F. Schilder, M. Hepple, G. Katz, and J. Pustejovsky. 2007. Semeval-2007 task 15: Tempeval temporal relation identification. In SemEval-2007. 